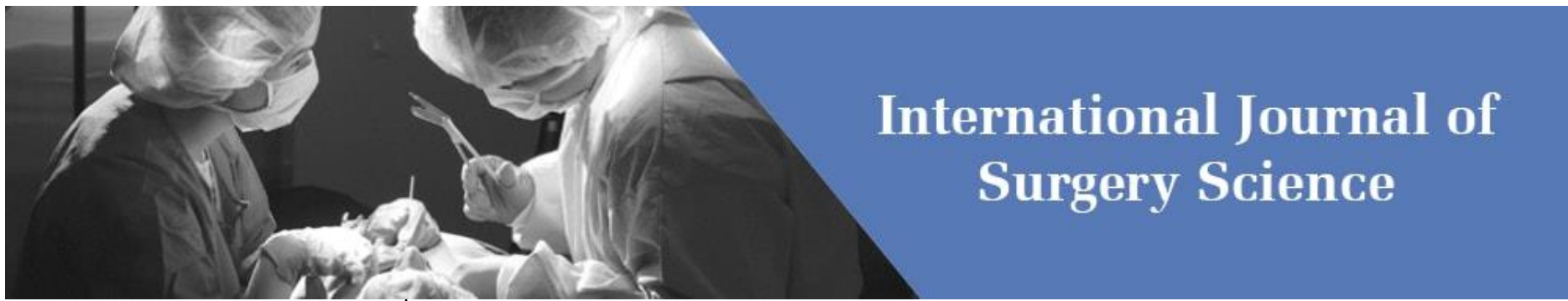

E-ISSN: 2616-3470

P-ISSN: 2616-3462

(C) Surgery Science www.surgeryscience.com

$2019 ; 3(4): 381-382$

Received: 07-08-2019

Accepted: 09-09-2019

Dr. Mezhuneituo Raleng

Tata Medical Center, Kolkata,

West Bengal, India

Dr. Anant Prakash Pore

Tata Medical Center, Kolkata,

West Bengal, India

Dr. RS Dahiya

PGIMS Rohtak,

Haryana, India
Corresponding Author:

Dr. Mezhuneituo Raleng

Tata Medical Center, Kolkata,

West Bengal, India

\section{Unexpected hidden knife blade in the chest wall: A rare case report}

\section{Dr. Mezhuneituo Raleng, Dr. Anant Prakash Pore and Dr. RS Dahiya}

DOI: https://doi.org/10.33545/surgery.2019.v3.i4g.271

Abstract

We report a 20-year-old boy presenting in OPD, after two and half month's history of assault and stab wound in left axilla with complaint of weakness and tingling in the left little and ring fingers. A knife blade retained in his chest wall was initially missed in emergency department. This case reminds us that, careful examination on all trauma patients is mandatory, especially those with penetrating injuries. Lack of apparent sign and symptoms, and a seemingly stable patient should not be an excuse for stopping further necessary assessment.

Keywords: Penetrating chest trauma, knife blade, retained, stab

\section{Introduction}

A 20-year-old, average built male presented to the outpatient after two and half month's history of alleged assault with complaints of weakness and tingling sensation over his left little and ring finger for fifteen days. On examination patient was afebrile, vitals were stable. He was conscious, oriented and no other neurological deficit could be elicited, other systemic examinations was normal. Patient had well healed scar about $3 \mathrm{~cm}$ in his left axilla with no local signs of inflammation. After a detailed history it unfold that the scar was due to a stab injury two and half months back (Fig. 1). Treatment was done in primary center, however detail reports were not available with the patient. On performing a chest X-ray we found a broken knife blade on his left upper chest with no signs of hemopneumothorax or signs of parenchymal injury (Fig. 2). After admitting him to the hospital a CT thorax was done, which confirmed our diagnosis, there was no evidence of vascular, thoracic injury, pleural, bony or cardiac injuries in the CT scan

After detailed investigations we posted him for surgery. The knife was lying over the left chest wall and had not penetrated the pleura, a simple extraction of the blade was fortunately possible. The post-operative period was uneventful and he was doing well after 6 months of follow up without any neurological deficit.

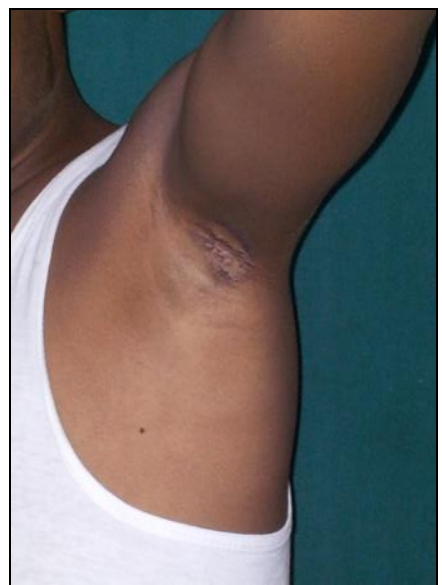

Fig 1: Healed scar wound seen on patient's axilla on external examination, at presentation to OPD 


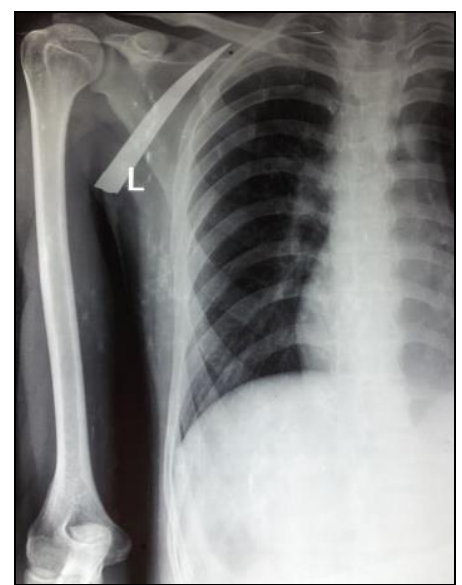

Fig 2: Chest X Ray (AP view) of left thorax showing a broken knife blade

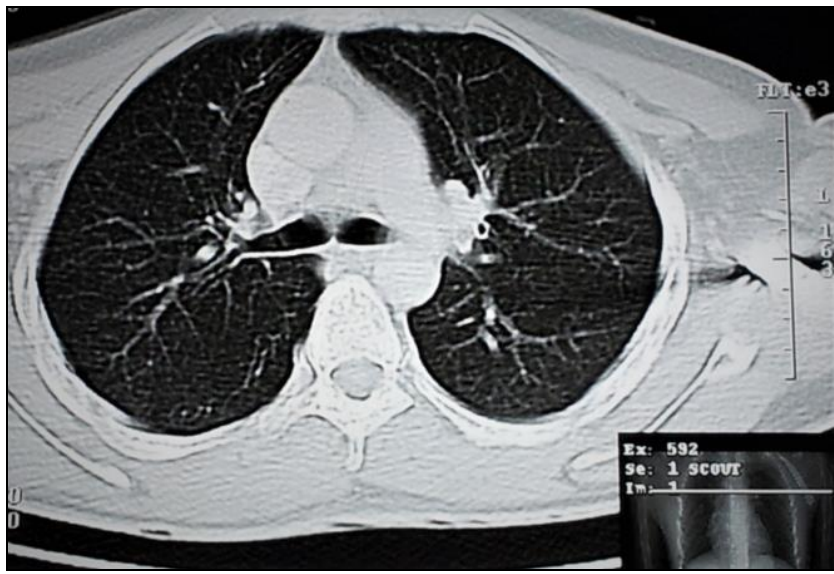

Fig 3: CT scan showing metallic density on the left upper thorax

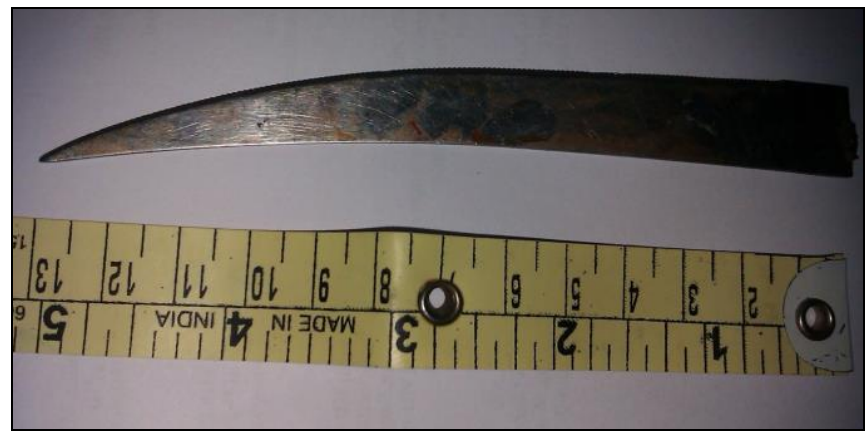

Fig 4: Extracted knife blade from the patients left thorax

\section{Discussion}

What's outstanding about this case is the late presentation and lack of symptoms, or maybe the apparent good fortune of the patient; as the foreign body did not cause him any major problems, considering the size of the kitchen knife. The sensible decision of the examining doctor in ordering for a chest X-ray instead of sending the patient to the neurologist is also noteworthy.

Clinical examination should be performed systematically; assessment of the extent and depth of the entry wound along with the surface anatomy can point to specific organ and neurovascular injuries. Detailed history from paramedics or witnesses can be helpful in determining whether the knife was intact after stabbing ${ }^{[1]}$.

Commonest cause of stab injuries requiring admission are in increasing order: the chest, abdomen and neck, however cardiac injuries are rare and incidence is highest in middle age males ${ }^{[2,3}$, 4]. The thorax has also been the site for most of the retained knife injuries ${ }^{[5]}$. Though previous studies have reported on retained knife blade, most of them have been in the emergency setting and patient reporting late after the incident have been quite rare. A retained foreign body carries several risks to the patient, they are highly susceptible to pyogenic infections, and the infections are resistant to antibiotic therapy, before the foreign bodies are removed ${ }^{[6]}$ they can also migrate to other areas and may cause life threatening complications and can manifest years after the trauma ${ }^{[7]}$. An abnormal delay in presentation is associated with adverse outcomes and can lead to mediastinal abscesses, empyema, aneurysm of major vessels, osteomyelitis, intraabdominal sepsis, and septicemia ${ }^{[9,10]}$.

This case report highlights the importance of routine radiographic examination for stab wounds so that misadventures may be avoided. Whenever in doubt one should always order a chest X-ray and if necessary a computed tomography (CT) scan, CT scans can give an array of information apart from the path of the object, organs involved and severity of injury ${ }^{[8]}$.

\section{Conclusion}

It is our recommendation that all chest trauma patients should undergo detailed clinical examination with a low threshold for imaging studies. Retained thoracic knife blades require proper preop planning and detailed investigation as they can give unexpected complications during surgery.

\section{References}

1. Hanoch J, Feigin E, Pikarsky A et al. Stab wound associated with terrorist attacks in Israel. JAMA. 1996; 276:388-390

2. Manojkumar S Nair, Mohammed $\mathrm{M}$ Uzzaman et al. Changing trends in the pattern and outcome of stab injuries at a North London hospital. J Emerg Trauma Shock. 2011; 4(4):455-60.

3. Gruen RL. Injury in indigenous communities: A call for enlightened action. ANZ J Surg. 2007; 77:930-1.

4. Jacob AO, Boseto F, Ollapallil J. Epidemic of stab injuries: An Alice Springs dilemma. ANZ J Surg. 2007; 77:621-5.

5. Sobnach S, Nicol A, Nathire H, Kahn D, Navsaria P. Management of the retained knife blade. World J Surg. 2010; 34(7):1648-52.

6. Kim TJ, Goo JM, Moon MH, Im JG, Kim MY. Foreign bodies in the chest: how come they are seen in adults? Korean J Radiol. 2001; 2:87-96.

7. Sokouti M, Montazeri V. Delayed massive hemoptysis 20 years after lung stabbing: an unusual presentation. Eur $\mathrm{J}$ Cardiothorac Surg. 2007; 32:679-8.

8. de Vries CS, Africa M, Gebremariam FA, van Rensburg JJ, Otto SF, Potgieter HF et al. The imaging of stab injuries. Acta Radiol. 2010; 51:92-106.

9. Madhok BM, Roy DD, Yeluri S. Penetrating arrow injuries in Western India. Injury. 2005; 36:1045-1050.

10. Fingleton LJ. Arrow wounds to the heart and mediastinum. Br J Surg. 1987; 74:126-128. 